\title{
SISTEMAS EM PARALELO COM DIFERENTES TIPOS DE TAXA DE RISCO: ANÁLISE DE PERÍODOS E DE CUSTOS DE INSPECÇÃO
}

\author{
J. Rodrigues Dias * \\ Paulo Infante \\ Departamento de Matemática \\ Universidade de Évora \\ Évora - Portugal \\ rodrigues-dias@clix.pt \\ * Corresponding author/autor para quem as correspondências devem ser encaminhadas \\ Recebido em 11/2001; aceito em 09/2004 após 1 revisão \\ Received November 2001; accepted September 2004 after one revision
}

\section{Resumo}

Considerem-se dois ou mais sistemas em paralelo com diferentes tipos de taxas de risco e cujo estado de bom ou mau funcionamento é apenas detectado através de inspecções perfeitas. Considerando diferentes custos de inspecção e de mau funcionamento por unidade de tempo, em relação a cada um dos sistemas, pretende-se analisar, na sequência de trabalhos anteriores, o período e o custo óptimos por ciclo do sistema global resultante, que apresenta maior fiabilidade, tomando como referência um dos sistemas iniciais em paralelo.

Palavras-chave: fiabilidade; período de inspecção; sistemas em paralelo.

\begin{abstract}
Let us consider two or more parallel systems with different hazard rates whose state of effective or noneffective functioning is only detected by perfect inspections. Considering different costs for each inspection and for unit of time of non-effective functioning, we analyse, following in the steps of previous works, the optimal inspection period and the respective expected total cost per cycle of the resulting global system which presents greater reliability, taking as reference one of the initial parallel systems.
\end{abstract}

Keywords: reliability; inspection period; parallel systems. 


\section{Introdução}

Considere-se um sistema cujo tempo de vida $\mathrm{T}$ é uma variável aleatória contínua com valor médio $\mathrm{E}(\mathrm{T})$, cujo estado de bom ou mau funcionamento é apenas conhecido se o sistema for inspeccionado. Admita-se, ainda, que existe um custo fixo $C_{1}$ por cada inspecção feita ao sistema e um custo fixo $\mathrm{C}_{2}$ por cada unidade de tempo de mau funcionamento não detectado. Considere-se, por outro lado, que as inspecções consideradas neste trabalho são periódicas, perfeitas, de duração nula e que não interferem no estado do sistema.

O problema que se nos coloca num esquema de inspecção periódica é determinar uma sequência de instantes de inspecção $\mathrm{P}$, com $\mathrm{i}=1,2,3, \ldots$, em que $\mathrm{P}$ é o período, de tal forma que seja mínimo o custo total médio por ciclo $\mathrm{E}(\mathrm{C})$ dado por:

$$
\mathrm{E}(\mathrm{C})=\mathrm{C}_{1} \mathrm{E}(\mathrm{N})+\mathrm{C}_{2} \mathrm{E}(\mathrm{D})
$$

em que $\mathrm{E}(\mathrm{N})$ e $\mathrm{E}(\mathrm{D})$ são, respectivamente, o número médio de inspecções e o tempo médio de detecção. Considera-se que um ciclo começa no instante inicial de funcionamento (pode ser após a reparação de uma falha, após a qual o sistema se considera como novo) e termina no instante em que a falha é detectada.

$E(N)$ e $E(D)$ admitem interpretações geométricas curiosas, permitindo conclusões interessantes (Rodrigues Dias, 1983, 1987). Em particular, note-se que

$$
\mathrm{E}(\mathrm{N})=\sum_{\mathrm{i}=0}^{\infty} \mathrm{R}(\mathrm{iP})
$$

onde $\mathrm{R}(\mathrm{t})$ representa a função de fiabilidade do sistema, definida por

$$
\mathrm{R}(\mathrm{t})=\mathrm{P}(\mathrm{T}>\mathrm{t})=1-\mathrm{F}(\mathrm{t})
$$

em que $\mathrm{F}(\mathrm{t})$ é a função de distribuição de $\mathrm{T}$. Note-se também que

$$
\mathrm{E}(\mathrm{D})=\mathrm{PE}(\mathrm{N})-\mathrm{E}(\mathrm{T})
$$

A solução para o período de inspecção $\mathrm{P}$ é obtida através da resolução da equação (Rodrigues Dias, 1983):

$$
\sum_{\mathrm{i}=0}^{\infty} \mathrm{R}(\mathrm{iP})-(\mathrm{r}+\mathrm{P}) \sum_{\mathrm{i}=1}^{\infty} \text { if }(\mathrm{iP})=0, \quad \mathrm{r}=\frac{\mathrm{C}_{1}}{\mathrm{C}_{2}}
$$

onde f(t) é a função contínua de densidade de $\mathrm{T}$.

Nakagawa \& Yasui (1979), admitindo que $E(D)=P / 2$, obtiveram a solução aproximada $P^{*}$ para o período de inspecção:

$$
\mathrm{P}^{*}=\sqrt{2 \mathrm{rE}(\mathrm{T})}
$$

Em Rodrigues Dias (1983) analisou-se, com detalhe e, em particular, em termos de custos, a validade desta aproximação para diferentes distribuições de tempos de vida e diferentes valores de $r$. Note-se que na solução não aparecem os valores individuais de $C_{1}$ e $C_{2}$ mas apenas o seu cociente $r$.

Em Rodrigues Dias (1990a) obteve-se uma outra aproximação para o período de inspecção $\mathrm{P}^{* *}$, dada por: 


$$
\mathrm{P}^{* *}=\frac{\mathrm{P}^{*}}{1+0.234 \sqrt{\mathrm{r}^{\prime}}}, \mathrm{r}^{\prime}=\frac{\mathrm{r}}{\mathrm{E}(\mathrm{T})}
$$

que pode ser considerada como uma generalização de $\mathrm{P}^{*}$. Em termos globais, concluiu-se que $\mathrm{P}^{* *}$ é melhor que $\mathrm{P}^{*} \mathrm{e}$, em especial para o caso da distribuição exponencial, pode ser considerada (quase) óptima.

Rodrigues Dias (2000a) mostrou, através de alguns exemplos, que quando se introduz um novo componente (sistema) em série num sistema já existente, ambos com tempo de vida exponencial, o período de inspecção óptimo do novo sistema pode aumentar ou diminuir, apesar da diminuição da fiabilidade do sistema global resultante, dependendo dos novos custos de inspecção e de mau funcionamento. Em Rodrigues Dias \& Infante (1999) generaliza-se esse estudo, considerando, em particular, tempos de vida com uma distribuição de Weibull com diferentes parâmetros de forma, obtendo, para cada $r$, uma relação entre os custos por forma a que o período quase-óptimo no sistema em série e no sistema inicial seja o mesmo. Em Rodrigues Dias \& Infante (2001) aprofunda-se o estudo feito, numa perspectiva de comparação de períodos e de custos totais médios por ciclo. Em Rodrigues Dias (2001) alarga-se o estudo ao caso em que se consideram componentes em paralelo, admitindo que o respectivo tempo de vida é exponencial.

O caso em que se consideram inspecções não periódicas tem também sido largamente tratado. Por exemplo, em Barlow \& Proschan (1965), Munford \& Shahani (1972) e Rodrigues Dias (1987, 1990b, 2000b), diversas situações são tratadas.

\section{Sistema com Componentes em Paralelo com Diferentes Tipos de Taxa de Risco}

Considere-se, então, que, em relação a um sistema inicial, se introduz um ou mais componentes em paralelo. Este caso é importante em situações em que o custo por unidade de tempo de mau funcionamento é relevante, como acontece, por exemplo, em contextos de saúde (hospitalares e outros), de segurança (de pessoas e bens), de telecomunicações e militares (de defesa e de ataque). Nestas situações, com o objectivo de aumentar a fiabilidade dos sistemas em causa, pode-se introduzir um ou mais componentes em paralelo, de sorte que o sistema resultante global funcione desde que um deles, pelo menos, funcione. Na Figura 1 representa-se tal situação, para o caso de dois componentes.

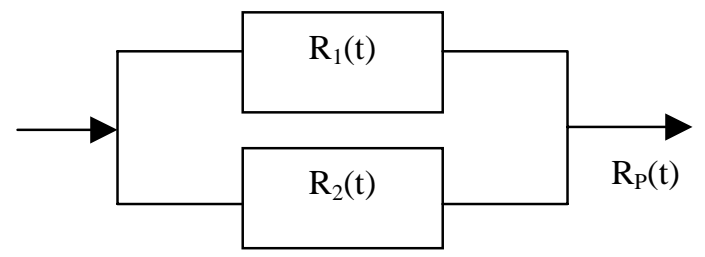

Figura 1 - Sistema com dois componentes em paralelo, com funções de fiabilidade $R_{1}(t)$ e $R_{2}(t)$, com função de fiabilidade global $\mathrm{R}_{\mathrm{P}}(\mathrm{t})$.

Seja, então, no caso de um sistema com dois componentes, $\mathrm{R}_{\mathrm{i}}(\mathrm{t})$ a função de fiabilidade do componente $\mathrm{i}(\mathrm{i}=1,2)$, com tempo de vida $\mathrm{T}_{\mathrm{i}}$. A função de fiabilidade do sistema resultante $\mathrm{R}_{\mathrm{P}}(\mathrm{t})$ passa a ser: 


$$
\begin{aligned}
R_{P}(t) & =R_{1}(t)+R_{2}(t)-R_{1}(t) R_{2}(t) \\
& =1-\left[1-R_{1}(t)\right]\left[1-R_{2}(t)\right]
\end{aligned}
$$

Repare-se que o resultado expresso em (8) resulta directamente da aplicação do teorema da probabilidade total.

Seja, ainda, $\mathrm{C}_{1}$ o custo de cada inspecção feita ao sistema inicial, quando este é apenas formado por um só componente e $\mathrm{C}_{1 \mathrm{P}} \mathrm{O}$ custo de cada inspecção do sistema global após ter sido introduzido o segundo componente. Poder-se-á, então, escrever:

$$
\mathrm{C}_{1 \mathrm{P}}=\mathrm{r}_{1} \cdot \mathrm{C}_{1}
$$

Analogamente, sendo $\mathrm{C}_{2}$ o custo por cada unidade de tempo de mau funcionamento não detectado do sistema inicial, quando este é apenas formado por um só componente e $\mathrm{C}_{2 \mathrm{P}} \mathrm{O}$ custo correspondente do sistema global após ter sido introduzido o segundo componente, ter-se-á:

$$
\mathrm{C}_{2 \mathrm{P}}=\mathrm{r}_{2} \cdot \mathrm{C}_{2}
$$

Seja:

$$
\mathrm{r}_{\mathrm{P}}=\mathrm{C}_{1 \mathrm{P}} / \mathrm{C}_{2 \mathrm{P}}=\left(\mathrm{r}_{1} / \mathrm{r}_{2}\right) \cdot\left(\mathrm{C}_{1} / \mathrm{C}_{2}\right)=\mathrm{kr}, \mathrm{k}=\mathrm{r}_{1} / \mathrm{r}_{2}
$$

Os valores de $r_{1}$ são, em princípio, maiores que 1. De facto, a inspecção global do sistema com os dois componentes terá um custo $C_{1 P}$ superior ao custo de inspecção $C_{1}$ do componente inicial. Note-se que se está aqui a admitir que quando o sistema é inspeccionado, ele o é na sua globalidade. Quanto aos valores de $r_{2}$, é natural supor que são iguais ou maiores que 1. Em particular, este último caso pode estar intimamente associado à necessidade de introduzir o segundo elemento em paralelo. Com base, então, nos valores possíveis de $\mathrm{r}_{1}$ e de $\mathrm{r}_{2}$, k poderá tomar qualquer valor positivo.

É fácil concluir que a fiabilidade do sistema global aumenta, daí resultando um tempo médio de vida superior a qualquer um dos componentes iniciais. E, sendo assim, poder-se-ia concluir que o período óptimo de inspecção aumentaria necessariamente. De resto, considerando como boa a solução aproximada dada por

$$
\mathrm{P}_{\mathrm{P}}^{*}=\sqrt{2 \mathrm{r}_{\mathrm{P}} \mathrm{E}\left(\mathrm{T}_{\mathrm{P}}\right)}
$$

e considerando, por outro lado, que, com $\mathrm{k} \geq 1$, se tem $\mathrm{r}_{\mathrm{p}} \geq \mathrm{r}$, poder-se-ia concluir de imediato que, como $\mathrm{E}\left(\mathrm{T}_{\mathrm{P}}\right)>\mathrm{E}\left(\mathrm{T}_{1}\right), \mathrm{P}_{\mathrm{P}}^{*}$ seria sempre maior que o período $\mathrm{P}^{*}{ }_{1}=\mathrm{P}^{*}$ que minimiza o custo total médio por ciclo quando se considera apenas o sistema inicial, com um componente. Note-se que, noutros trabalhos, com sistemas em série, se considerou sempre $\mathrm{k}>1$.

Apenas quando a relação dos custos diminuir de uma forma clara é que poderá verificar-se o caso oposto. Podendo tal acontecer em situações de interesse prático, com componentes em paralelo, este caso será aqui considerado.

Tratando-se de três ou mais componentes, a situação não se altera em termos qualitativos, complicando-se apenas ao nível analítico e computacional. Alguns resultados podem ser encontrados em Almeida (1998).

Neste artigo, vamos apresentar e analisar alguns resultados, considerando sistemas com um, dois e três componentes em paralelo, cada um deles com distribuição de tempo de vida 
Weibull, com diferentes valores do parâmetro de forma $\beta$. Procuramos, assim, estudar sistemas com componentes com diferentes tipos de taxa de risco $\mathrm{h}(\mathrm{t})$, dada por:

$$
\mathrm{h}(\mathrm{t})=\mathrm{f}(\mathrm{t}) / \mathrm{R}(\mathrm{t})
$$

Acentue-se que a distribuição de Weibull é largamente considerada neste contexto de fiabilidade, tendo a sua função densidade de probabilidade a expressão:

$$
\mathrm{f}(\mathrm{t})=\frac{\beta}{\alpha}\left(\frac{\mathrm{t}}{\alpha}\right)^{\beta-1}\left\{\exp \left[-\left(\frac{\mathrm{t}}{\alpha}\right)^{\beta}\right]\right\}
$$

em que $\beta$ é o parâmetro de forma e $\alpha$ o parâmetro de escala. Quando $\beta<1$ a taxa de risco h(t) é decrescente, quando $\beta=1$ a taxa de risco é constante e, finalmente, quando $\beta>1$, ela é crescente. Os dois últimos casos referidos são aqueles que, na prática, têm maior interesse.

Refira-se, a propósito, que é também esta distribuição de Weibull, com um parâmetro de forma $\beta=2.5$, que é usada para modelar o período de incubação do vírus HIV1 (Brookmeyer \& Goedert (1989) e Amaral et al. (2000)).

\section{Resultados Numéricos Obtidos e sua Análise}

Para efeitos de obtenção de resultados, considerou-se que $\mathrm{E}\left(\mathrm{T}_{\mathrm{i}}\right)=1$, pelo que o parâmetro de escala $\alpha$ foi convenientemente escolhido para cada valor do parâmetro de forma $ß$. Resultados idênticos poderiam ser obtidos para valores iguais de $\mathrm{r} / \mathrm{E}(\mathrm{T})$, tendo em conta que o custo total médio por ciclo E(C) é dado por (Rodrigues Dias, 1983):

$$
\mathrm{E}(\mathrm{C})=\mathrm{C}_{2} \mathrm{E}(\mathrm{T})\{[\mathrm{r} / \mathrm{E}(\mathrm{T})+\mathrm{P} / \mathrm{E}(\mathrm{T})] \mathrm{E}(\mathrm{N})-1\}
$$

Os resultados numéricos que a seguir se apresentam foram obtidos recorrendo a técnicas de simulação e de análise numérica. Note-se que a solução da equação (5) não pode ser obtida por métodos analíticos, já que, em particular, não podem ser obtidas as somas das séries envolvidas. De facto, basta ter em conta que a função de fiabilidade do sistema com (apenas) dois componentes em paralelo é dada, no caso dos seus tempos de vida terem uma distribuição de Weibull, pela expressão:

$$
\mathrm{R}_{\mathrm{P}}(\mathrm{t})=1-\left\{1-\exp \left[-\left(\frac{\mathrm{t}}{\alpha_{1}}\right)^{\beta_{1}}\right]\right\}\left\{1-\exp \left[-\left(\frac{\mathrm{t}}{\alpha_{2}}\right)^{\beta 2}\right]\right\}
$$

Como anteriormente, vamos considerar a solução exacta para o período de inspecção do sistema global, que designamos por $\mathrm{P}_{\mathrm{P}}$, e as duas soluções aproximadas $\mathrm{P}_{\mathrm{P}}^{*}$ e $\mathrm{P}_{\mathrm{P}}^{* *}$ dadas por:

$$
\begin{aligned}
& \mathrm{P}^{*}{ }_{\mathrm{P}}=\sqrt{2 \mathrm{r}_{\mathrm{P}} \mathrm{E}\left(\mathrm{T}_{\mathrm{P}}\right)} \\
& \mathrm{P}^{* *}{ }_{\mathrm{P}}=\mathrm{P}_{\mathrm{P}}^{*} /\left[1+.234 \mathrm{r}^{, 1 / 2}\right], \quad \mathrm{r}^{\prime}=\mathrm{r}_{\mathrm{P}} / \mathrm{E}\left(\mathrm{T}_{\mathrm{P}}\right)
\end{aligned}
$$

Considerando, neste momento, a solução $\mathrm{P}_{\mathrm{P}}{ }_{\mathrm{P}}$ como boa, verifica-se facilmente, a partir dela, que o período de inspecção do sistema em paralelo é maior que o valor correspondente do componente inicial, desde que $\mathrm{k}>1$. De facto, sendo $\mathrm{E}\left(\mathrm{T}_{\mathrm{P}}\right)>\mathrm{E}\left(\mathrm{T}_{1}\right)$, conforme antes se viu, com $\mathrm{k}>1$, tem-se $\mathrm{r}_{\mathrm{P}}>\mathrm{r}$ e, portanto, $\mathrm{P}_{\mathrm{P}}>\mathrm{P}_{1}$.

Uma questão que, então, pode ser colocada é verificar se existem valores de k (e em que condições, em caso afirmativo) que conduzam à situação oposta. No Quadro 1 apresentam-se 
resultados relativos a dois sistemas: num deles considera-se que o primeiro componente tem uma taxa de risco constante $\left(\beta_{1}=1\right)$ e o segundo componente uma taxa de risco crescente $\left(\beta_{2}=2\right)$; no outro sistema considera-se que ambos os componentes apresentam taxas de risco crescente $\left(\beta_{1}=2\right.$ e $\left.\beta_{2}=4\right)$.

Quadro 1 - Valores do período de inspecção para diferentes valores de r, no caso de dois sistemas com dois componentes em paralelo, considerando diferentes valores de $\mathrm{k}$.

\begin{tabular}{|c|c|c|c|c|c|c|c|c|}
\hline \multirow{3}{*}{$\mathbf{r}$} & \multicolumn{4}{|c|}{$\beta_{1}=1 ; \beta_{2}=2$} & \multicolumn{4}{|c|}{$\beta_{1}=2 ; \beta_{2}=4$} \\
\hline & \multirow{2}{*}{$\mathbf{P}_{1}$} & \multicolumn{3}{|c|}{$\mathbf{P}_{\mathbf{P}}$} & \multirow{2}{*}{$\mathbf{P}_{1}$} & \multicolumn{3}{|c|}{$\mathbf{P}_{\mathbf{P}}$} \\
\hline & & $k=.6$ & $k=.8$ & $k=1$ & & $k=.7$ & $k=.8$ & $k=1$ \\
\hline .001 & .0447 & .0412 & .0476 & .0532 & .0447 & .0416 & .0445 & .0497 \\
\hline .01 & .1382 & .1304 & .1505 & .1683 & .1414 & .1316 & .1407 & .1573 \\
\hline .1 & .4162 & .4131 & .4775 & .5347 & .4472 & .4178 & .4464 & .4883 \\
\hline
\end{tabular}

Verifica-se, claramente, que os resultados dependem da distribuição do tempo de vida do componente inicial e, naturalmente, do sistema global. Em particular, para $\mathrm{k}=.8$ e para os valores de $r$ apresentados, verifica-se que no primeiro sistema $\left(\beta_{1}=1\right.$ e $\left.\beta_{2}=2\right)$ se tem $P_{1}<P_{P}$, enquanto que no outro se verifica a situação oposta. Por outro lado, constata-se ainda que no primeiro sistema, ao passar do valor $\mathrm{k}=.6$ para $\mathrm{k}=.8$, o período de inspecção óptimo no sistema global passa de menor para maior, tomando como referência o valor correspondente do componente inicial. Idêntica situação se verifica no caso do segundo sistema $\left(\beta_{1}=2\right.$ e $\beta_{2}=4$ ), quando se considerou $\mathrm{k}=.8$ e $\mathrm{k}=1$.

Os factos antes referidos levam-nos a concluir que existe um valor de $\mathrm{k}$, menor que um, que conduz à igualdade $\mathrm{P}_{1}=\mathrm{P}_{\mathrm{P}}$. Em termos aproximados, poder-se-á escrever, com base nas expressões (6) e (18):

$$
\begin{aligned}
\mathrm{P}^{*}{ }_{1} / \mathrm{P}_{\mathrm{P}}{ }^{*} & =\left[2 \mathrm{rE}\left(\mathrm{T}_{1}\right)\right]^{1 / 2} /\left[2 \mathrm{krE}\left(\mathrm{T}_{\mathrm{P}}\right)\right]^{1 / 2} \\
& =\left[\mathrm{E}\left(\mathrm{T}_{1}\right) / \mathrm{kE}\left(\mathrm{T}_{\mathrm{P}}\right)\right]^{1 / 2}
\end{aligned}
$$

Considerando $\mathrm{P}^{*}=\mathrm{P}_{\mathrm{P}}{ }^{*}$, poder-se-ia escrever para solução aproximada de k:

$$
\mathrm{k}=\mathrm{E}\left(\mathrm{T}_{1}\right) / \mathrm{E}\left(\mathrm{T}_{\mathrm{P}}\right)
$$

Como é evidente, $\mathrm{k}$ depende dos componentes em paralelo, já que os tempos médios de vida também dependem.

Note-se que esta aproximação para k, apesar da sua simplicidade, não foi antes apresentada noutros trabalhos. Será interessante analisar quão válida ela é, quer no caso de componentes em paralelo, quer no caso de componentes em série.

À semelhança do que se fez em Rodrigues Dias (1983, 2001), analisemos agora as grandezas $\mathrm{Q}_{1}, \mathrm{Q}_{2}$ e $\mathrm{Q}_{3}$, dadas pelas expressões:

$$
\begin{aligned}
& \mathrm{Q}_{1}=[\mathrm{P} / 2-\mathrm{E}(\mathrm{D})] / \mathrm{E}(\mathrm{D}) \\
& \mathrm{Q}_{2}=\left[\mathrm{P}_{\mathrm{P}}^{*}-\mathrm{P}\right] / \mathrm{P} \\
& \mathrm{Q}_{3}=\left[\mathrm{P}^{* *}{ }_{\mathrm{P}}-\mathrm{P}\right] / \mathrm{P}
\end{aligned}
$$

Nos Quadros 2, 3, 4 e 5 estão apresentados, em percentagem, os valores obtidos, para sistemas diferentes, com $n$ componentes $(n=1,2$ e 3$)$ e para diferentes valores de $r(r=.001, .01$ e .1$)$. 
Quadro 2 - Valores de $\mathrm{Q}_{1}, \mathrm{Q}_{2}$ e $\mathrm{Q}_{3}$, em $\%$, para diferentes valores de r, considerando sistemas com $n$ componentes em paralelo $\left(\beta_{1}=.7 ; \beta_{2}=.8 ; \beta_{3}=1\right)$.

\begin{tabular}{|c|c|c|c|c|c|c|c|c|c|}
\hline & \multicolumn{3}{|c|}{$\mathbf{r = . 0 0 1}$} & \multicolumn{3}{c|}{$\mathbf{r}=. \mathbf{0 1}$} & \multicolumn{3}{c|}{$\mathbf{r}=\mathbf{. 1}$} \\
\cline { 2 - 10 } $\mathbf{n}$ & $\mathbf{Q}_{\mathbf{1}}$ & $\mathbf{Q}_{\mathbf{2}}$ & $\mathbf{Q}_{\mathbf{3}}$ & $\mathbf{Q}_{\mathbf{1}}$ & $\mathbf{Q}_{\mathbf{2}}$ & $\mathbf{Q}_{\mathbf{3}}$ & $\mathbf{Q}_{\mathbf{1}}$ & $\mathbf{Q}_{\mathbf{2}}$ & $\mathbf{Q}_{\mathbf{3}}$ \\
\hline $\mathbf{1}$ & -3.64 & 3.17 & 2.41 & -7.49 & 6.76 & 4.32 & -14.26 & 14.07 & 6.21 \\
\hline $\mathbf{2}$ & -1.58 & .10 & -.48 & -1.00 & .67 & -1.16 & -2.93 & 3.88 & -1.85 \\
\hline $\mathbf{3}$ & -3.05 & -.03 & -.55 & -.96 & -.07 & -1.70 & -.14 & -.15 & -5.15 \\
\hline
\end{tabular}

Quadro 3 - Valores de $\mathrm{Q}_{1}, \mathrm{Q}_{2}$ e $\mathrm{Q}_{3}$, em $\%$, para diferentes valores de $\mathrm{r}$, considerando sistemas com $n$ componentes em paralelo $\left(\beta_{1}=1 ; \beta_{2}=1 ; \beta_{3}=1\right)$.

\begin{tabular}{|c|c|c|c|c|c|c|c|c|c|}
\hline & \multicolumn{3}{|c|}{$\mathbf{r}=\mathbf{. 0 0 1}$} & \multicolumn{3}{c|}{$\mathbf{r = . 0 1}$} & \multicolumn{3}{c|}{$\mathbf{r}=. \mathbf{1}$} \\
\cline { 2 - 10 } $\mathbf{n}$ & $\mathbf{Q}_{\mathbf{1}}$ & $\mathbf{Q}_{\mathbf{2}}$ & $\mathbf{Q}_{\mathbf{3}}$ & $\mathbf{Q}_{\mathbf{1}}$ & $\mathbf{Q}_{\mathbf{2}}$ & $\mathbf{Q}_{\mathbf{3}}$ & $\mathbf{Q}_{\mathbf{1}}$ & $\mathbf{Q}_{\mathbf{2}}$ & $\mathbf{Q}_{\mathbf{3}}$ \\
\hline $\mathbf{1}$ & -.73 & .74 & .00 & -2.25 & 2.36 & .02 & -6.47 & 7.45 & .04 \\
\hline $\mathbf{2}$ & .00 & .00 & -.60 & -.01 & .02 & -1.86 & -.26 & .58 & -5.15 \\
\hline $\mathbf{3}$ & .00 & -.00 & -.54 & .01 & -.02 & -1.72 & .30 & -.60 & -5.75 \\
\hline
\end{tabular}

Quadro 4 - Valores de $\mathrm{Q}_{1}, \mathrm{Q}_{2}$ e $\mathrm{Q}_{3}$, em $\%$, para diferentes valores de r, considerando sistemas com n componentes em paralelo $\left(\beta_{1}=1 ; \beta_{2}=2 ; \beta_{3}=1\right)$.

\begin{tabular}{|c|c|c|c|c|c|c|c|c|c|}
\hline & \multicolumn{3}{|c|}{$\mathbf{r = . 0 0 1}$} & \multicolumn{3}{c|}{$\mathbf{r}=. \mathbf{0 1}$} & \multicolumn{3}{c|}{$\mathbf{r}=\mathbf{. 1}$} \\
\cline { 2 - 10 } $\mathbf{n}$ & $\mathbf{Q}_{\mathbf{1}}$ & $\mathbf{Q}_{\mathbf{2}}$ & $\mathbf{Q}_{\mathbf{3}}$ & $\mathbf{Q}_{\mathbf{1}}$ & $\mathbf{Q}_{\mathbf{2}}$ & $\mathbf{Q}_{\mathbf{3}}$ & $\mathbf{Q}_{\mathbf{1}}$ & $\mathbf{Q}_{\mathbf{2}}$ & $\mathbf{Q}_{\mathbf{3}}$ \\
\hline $\mathbf{1}$ & -.73 & .74 & .00 & -2.25 & 2.36 & .02 & -6.47 & 7.45 & .04 \\
\hline $\mathbf{2}$ & .42 & .00 & -.61 & .14 & -.01 & -1.94 & .25 & -.48 & -6.30 \\
\hline $\mathbf{3}$ & -.38 & .00 & -.56 & -.12 & .00 & -1.75 & .01 & -.17 & -5.49 \\
\hline
\end{tabular}

Quadro 5 - Valores de $\mathrm{Q}_{1}, \mathrm{Q}_{2}$ e $\mathrm{Q}_{3}$, em $\%$, para diferentes valores de r, considerando sistemas com $n$ componentes em paralelo $\left(\beta_{1}=1 ; \beta_{2}=2 ; \beta_{3}=2\right)$.

\begin{tabular}{|c|c|c|c|c|c|c|c|c|c|}
\hline & \multicolumn{3}{|c|}{$\mathbf{r}=\mathbf{0 0 1}$} & \multicolumn{3}{c|}{$\mathbf{r}=. \mathbf{0 1}$} & \multicolumn{3}{c|}{$\mathbf{r}=\mathbf{. 1}$} \\
\cline { 2 - 10 } $\mathbf{n}$ & $\mathbf{Q}_{\mathbf{1}}$ & $\mathbf{Q}_{\mathbf{2}}$ & $\mathbf{Q}_{\mathbf{3}}$ & $\mathbf{Q}_{\mathbf{1}}$ & $\mathbf{Q}_{\mathbf{2}}$ & $\mathbf{Q}_{\mathbf{3}}$ & $\mathbf{Q}_{\mathbf{1}}$ & $\mathbf{Q}_{\mathbf{2}}$ & $\mathbf{Q}_{\mathbf{3}}$ \\
\hline $\mathbf{1}$ & -.73 & .74 & .00 & -2.25 & 2.36 & .02 & -6.47 & 7.45 & .04 \\
\hline $\mathbf{2}$ & .42 & .00 & -.61 & .14 & -.01 & -1.94 & .25 & -.48 & -6.30 \\
\hline $\mathbf{3}$ & -.27 & .00 & -.58 & -.08 & .00 & -1.82 & -.06 & .13 & -5.40 \\
\hline
\end{tabular}

Os resultados obtidos permitem-nos, entre outras, fazer as seguintes considerações:

a) Os valores de $\mathrm{Q}_{1}$, em módulo, apenas com duas excepções, diminuem com $\mathrm{n}$. Este facto significa que, à medida que $\mathrm{n}$ aumenta, o tempo médio de detecção se aproxima, de um modo geral, de metade do período de inspecção.

b) Os valores de $Q_{2}$ diminuem com $n$, o que é uma consequência da conclusão expressa em a). No entanto, poderá acontecer que $\mathrm{Q}_{2}$ diminua mesmo quando $\mathrm{Q}_{1}$ aumente, o que tem a ver com as distribuições dos tempos de vida e com os valores das diversas grandezas intervenientes. 
c) Quando $\mathrm{r}$ aumenta, tipicamente, os valores de $\mathrm{Q}_{1}, \mathrm{Q}_{2}$ e $\mathrm{Q}_{3}$ aumentam, o que está de acordo com interpretações e resultados anteriores (Rodrigues Dias, 1983, 1987).

d) Para $\mathrm{n}=1$ ( sistema com um só componente), verifica-se, nos casos apresentados, que $\mathrm{Q}_{2}>\mathrm{Q}_{3}$. Esta constatação leva-nos aqui a concluir que a aproximação $\mathrm{P}^{* *}{ }_{\mathrm{P}}$ é melhor que $\mathrm{P}_{\mathrm{P}}^{*}$. No entanto, tal nem sempre acontece com outras distribuições de tempos de vida (Rodrigues Dias, 1983).

e) Para $n>1$, verifica-se quase sempre a situação oposta da referida no ponto anterior. Neste caso, a aproximação $\mathrm{P}^{* *}{ }_{\mathrm{P}}$ é pior que $\mathrm{P}_{\mathrm{P}}^{*}$.

f) Em jeito de síntese, poder-se-á dizer que, para $n>1$ e para $r=.001$ e $r=.01$, os erros relativos das aproximações $\mathrm{P}_{\mathrm{P}}^{*}$ e $\mathrm{P}^{* *}{ }_{\mathrm{P}}$ são inferiores (em módulo) a $2 \%$, pelo que, tendo em conta a fraca sensibilidade do custo total médio por ciclo, conforme se verá depois, se podem considerar como sendo quase óptimas.

Nos Quadros 6, 7, 8 e 9, análogos a Quadros anteriores, apresentam-se agora os valores das grandezas:

$$
\begin{aligned}
& \mathrm{Q}_{4}=\left[\mathrm{E}^{*}\left(\mathrm{C}_{\mathrm{P}}\right)-\mathrm{E}\left(\mathrm{C}_{\mathrm{P}}\right)\right] / \mathrm{E}\left(\mathrm{C}_{\mathrm{P}}\right) \\
& \mathrm{Q}_{5}=\left[\mathrm{E}^{* *}\left(\mathrm{C}_{\mathrm{P}}\right)-\mathrm{E}\left(\mathrm{C}_{\mathrm{P}}\right)\right] / \mathrm{E}\left(\mathrm{C}_{\mathrm{P}}\right)
\end{aligned}
$$

A partir destes Quadros, cujos valores, em percentagem, foram calculados considerando $\mathrm{C}_{2}=100$, é possível acentuar, entre outros, os resultados seguintes:

a) Quando $n=1$, verifica-se que $\mathrm{Q}_{4}>\mathrm{Q}_{5}$, o que significa que a aproximação $\mathrm{P}_{1}{ }_{1}$ é pior que $\mathrm{P}^{* *}$.

b) Para $\mathrm{n}>1$, verifica-se quase sempre que $\mathrm{Q}_{4}<\mathrm{Q}_{5}$, o que significa que a aproximação $\mathrm{P}_{\mathrm{P}}^{*}$ é melhor que $\mathrm{P}^{* *}$. Este resultado e o anterior, em função de conclusões antecedentes, seriam de esperar.

c) Quando n aumenta, $\mathrm{Q}_{4}$ diminui ou, pelo menos, não aumenta. Em particular, para $\mathrm{n}=3$, verifica-se que é quase nulo $\left(\mathrm{Q}_{4} \cong .000 \%\right)$, pelo que se pode afirmar que, neste caso, a aproximação correspondente do período de inspecção se pode considerar como (quase) óptima.

d) Finalmente, comparando os erros relativos das aproximações dos períodos de inspecção com os dos custos associados, é óbvio que estes são incomparavelmente inferiores, sendo muito próximos de zero, pelo que aquelas aproximações dos períodos de inspecção, em termos práticos, têm uma extrema relevância.

Considere-se, por fim, no Quadro 10, para os mesmos sistemas e valores, a grandeza $\mathrm{Q}_{6}$, dada por:

$$
\mathrm{Q}_{6}=\mathrm{E}\left(\mathrm{C}_{\mathrm{P}}\right) /\left[\mathrm{E}\left(\mathrm{T}_{\mathrm{P}}\right)+\mathrm{E}\left(\mathrm{D}_{\mathrm{P}}\right)\right]
$$

em que o denominador representa a duração média de um ciclo. Recorde-se que $E\left(C_{P}\right)$ é calculado, para cada sistema e para cada valor de $\mathrm{r}$, com base no respectivo tempo médio de vida $\mathrm{E}\left(\mathrm{T}_{\mathrm{P}}\right)$. Os valores de $\mathrm{Q}_{6}$ têm o significado de um custo por unidade de tempo.

A partir dos valores obtidos, não esquecendo que r/E(T) varia de sistema para sistema, pode constatar-se o seguinte: 
a) Para cada valor de $\mathrm{n}$, à medida que a taxa de risco do sistema global vai aumentando, no sentido de a respectiva função de fiabilidade se aproximar de uma forma rectangular (função densidade de probabilidade de Dirac), verifica-se que $E\left(N_{P}\right)$ vai diminuindo, tendendo para 1. De igual modo, $\mathrm{E}\left(\mathrm{D}_{\mathrm{P}}\right)$ também diminui, tendendo para 0 .

b) Com base nas considerações do ponto anterior, para cada um dos valores de n, o custo total médio mínimo por ciclo $\mathrm{E}\left(\mathrm{C}_{\mathrm{P}}\right)$ também diminui, tendendo, no limite, para $\mathrm{E}\left(\mathrm{C}_{1}\right)$.

c) De igual modo, para cada $\mathrm{n}, \mathrm{E}\left(\mathrm{T}_{\mathrm{P}}\right)$ vai diminuindo à medida que a taxa de risco dos componentes vai aumentando, tendendo para o valor comum dos tempos médios de vida dos componentes $\mathrm{E}\left(\mathrm{T}_{\mathrm{i}}\right)$, ou para o maior deles.

d) Quando a um dado sistema se acrescenta um novo componente em paralelo, $\mathrm{E}\left(\mathrm{T}_{\mathrm{P}}\right)$ aumenta, conforme se referiu logo no início.

e) De igual modo, $\mathrm{E}\left(\mathrm{C}_{\mathrm{P}}\right)$ aumenta quando $\mathrm{n}$ aumenta.

f) Quando $n=1, Q_{6}$ diminui quando $\beta$ aumenta. Repare-se que $E\left(C_{1}\right)$ diminui mas diminui também $\mathrm{E}\left(\mathrm{D}_{1}\right)$, estando a considerar-se que $\mathrm{E}\left(\mathrm{T}_{1}\right)=1$.

Quando $\mathrm{n}>1, \mathrm{Q}_{6}$ aumenta quando a taxa de risco aumenta $(\mathrm{R}(\mathrm{t})$ aproximando-se de uma forma rectangular). Neste caso, verifica-se que $\mathrm{E}\left(\mathrm{C}_{\mathrm{P}}\right)$ diminui, mas diminui de uma forma mais acentuada $\mathrm{E}\left(\mathrm{T}_{\mathrm{P}}\right)$. Constata-se ainda que $\mathrm{E}\left(\mathrm{D}_{\mathrm{P}}\right)$ diminui, embora de uma forma ligeira.

Quadro 6 - Valores de $\mathrm{Q}_{4}$ e $\mathrm{Q}_{5}$, em \%, para diferentes valores de r, considerando sistemas com $\mathrm{n}$ componentes em paralelo $\left(\beta_{1}=.7 ; \beta_{2}=.8 ; \beta_{3}=1\right)$.

\begin{tabular}{|c|c|c|c|c|c|c|}
\hline \multirow{2}{*}{$\mathbf{n}$} & \multicolumn{2}{|c|}{$\mathbf{r = . 0 0 1}$} & \multicolumn{2}{c|}{$\mathbf{r}=. \mathbf{0 1}$} & \multicolumn{2}{c|}{$\mathbf{r}=\mathbf{. 1}$} \\
\cline { 2 - 7 } & $\mathbf{Q}_{\mathbf{4}}$ & $\mathbf{Q}_{\mathbf{5}}$ & $\mathbf{Q}_{\mathbf{4}}$ & $\mathbf{Q}_{\mathbf{5}}$ & $\mathbf{Q}_{\mathbf{4}}$ & $\mathbf{Q}_{\mathbf{5}}$ \\
\hline $\mathbf{1}$ & .050 & .029 & .221 & .092 & .873 & .182 \\
\hline $\mathbf{2}$ & .000 & .001 & .002 & .007 & .072 & .017 \\
\hline $\mathbf{3}$ & .000 & .001 & .000 & .014 & .000 & .130 \\
\hline
\end{tabular}

Quadro 7 - Valores de $\mathrm{Q}_{4}$ e $\mathrm{Q}_{5}$, em \%, para diferentes valores de $\mathrm{r}$, considerando sistemas com $\mathrm{n}$ componentes em paralelo $\left(\beta_{1}=1 ; \beta_{2}=1 ; \beta_{3}=1\right)$.

\begin{tabular}{|c|c|c|c|c|c|c|}
\hline \multirow{2}{*}{$\mathbf{n}$} & \multicolumn{2}{|c|}{$\mathbf{r}=\mathbf{. 0 0 1}$} & \multicolumn{2}{c|}{$\mathbf{r = . 0 1}$} & \multicolumn{2}{c|}{$\mathbf{r = . 1}$} \\
\cline { 2 - 7 } & $\mathbf{Q}_{\mathbf{4}}$ & $\mathbf{Q}_{\mathbf{5}}$ & $\mathbf{Q}_{\mathbf{4}}$ & $\mathbf{Q}_{\mathbf{5}}$ & $\mathbf{Q}_{\mathbf{4}}$ & $\mathbf{Q}_{\mathbf{5}}$ \\
\hline $\mathbf{1}$ & .003 & .000 & .027 & .000 & .255 & .000 \\
\hline $\mathbf{2}$ & .000 & .002 & .000 & .017 & .002 & .131 \\
\hline $\mathbf{3}$ & .000 & .001 & .000 & .015 & .002 & .159 \\
\hline
\end{tabular}

Quadro 8 - Valores de $\mathrm{Q}_{4}$ e $\mathrm{Q}_{5}$, em \%, para diferentes valores de $\mathrm{r}$, considerando sistemas com n componentes em paralelo $\left(\beta_{1}=1 ; \beta_{2}=2 ; \beta_{3}=1\right)$.

\begin{tabular}{|c|c|c|c|c|c|c|}
\hline \multirow{2}{*}{$\mathbf{n}$} & \multicolumn{2}{|c|}{$\mathbf{r}=. \mathbf{0 0 1}$} & \multicolumn{2}{c|}{$\mathbf{r}=. \mathbf{0 1}$} & \multicolumn{2}{c|}{$\mathbf{r}=. \mathbf{1}$} \\
\cline { 2 - 7 } & $\mathbf{Q}_{\mathbf{4}}$ & $\mathbf{Q}_{\mathbf{5}}$ & $\mathbf{Q}_{\mathbf{4}}$ & $\mathbf{Q}_{\mathbf{5}}$ & $\mathbf{Q}_{\mathbf{4}}$ & $\mathbf{Q}_{\mathbf{5}}$ \\
\hline $\mathbf{1}$ & .003 & .000 & .027 & .000 & .255 & .000 \\
\hline $\mathbf{2}$ & .000 & .002 & .000 & .019 & .001 & .191 \\
\hline $\mathbf{3}$ & .000 & .002 & .000 & .015 & .000 & .146 \\
\hline
\end{tabular}


Quadro 9 - Valores de $\mathrm{Q}_{4}$ e $\mathrm{Q}_{5}$, em \%, para diferentes valores de r, considerando sistemas com $\mathrm{n}$ componentes em paralelo $\left(\beta_{1}=1 ; \beta_{2}=2 ; \beta_{3}=2\right)$.

\begin{tabular}{|c|c|c|c|c|c|c|}
\hline \multirow{2}{*}{$\mathbf{n}$} & \multicolumn{2}{|c|}{$\mathbf{r = . 0 0 1}$} & \multicolumn{2}{c|}{$\mathbf{r = . 0 1}$} & \multicolumn{2}{c|}{$\mathbf{r = . 1}$} \\
\cline { 2 - 7 } & $\mathbf{Q}_{4}$ & $\mathbf{Q}_{5}$ & $\mathbf{Q}_{4}$ & $\mathbf{Q}_{5}$ & $\mathbf{Q}_{4}$ & $\mathbf{Q}_{5}$ \\
\hline $\mathbf{1}$ & .003 & .000 & .027 & .000 & .255 & .000 \\
\hline $\mathbf{2}$ & .000 & .002 & .000 & .019 & .001 & .191 \\
\hline $\mathbf{3}$ & .000 & .002 & .000 & .016 & .000 & .143 \\
\hline
\end{tabular}

Quadro 10 - Valores de $E\left(T_{P}\right), E\left(D_{P}\right), E\left(N_{P}\right), E\left(C_{P}\right)$ e $Q_{6}$, para diferentes valores de r e diferentes sistemas.

\begin{tabular}{|c|c|c|c|c|c|c|c|}
\hline $\mathbf{n}$ & Componentes & $\mathbf{r}$ & $\mathbf{E}\left(\mathbf{T}_{\mathbf{P}}\right)$ & $\mathbf{E}\left(\mathbf{D}_{\mathbf{P}}\right)$ & $\mathbf{E}\left(\mathbf{N}_{\mathbf{P}}\right)$ & $\mathbf{E}\left(\mathbf{C}_{\mathbf{P}}\right)$ & $\mathbf{Q}_{6}$ \\
\hline \multirow{9}{*}{1} & \multirow{3}{*}{$\beta=0.7$} & .001 & 1.000 & .022 & 23.59 & 4.61 & 4.51 \\
\hline & & .01 & 1.000 & .072 & 8.09 & 15.25 & 14.23 \\
\hline & & .1 & 1.000 & .229 & 3.13 & 54.20 & 44.12 \\
\hline & \multirow{3}{*}{$\beta=1$} & .001 & 1.000 & .022 & 23.03 & 4.54 & 4.44 \\
\hline & & .01 & 1.000 & .071 & 7.75 & 14.82 & 13.84 \\
\hline & & .1 & 1.000 & .223 & 2.94 & 51.62 & 42.23 \\
\hline & \multirow{3}{*}{$\beta=2$} & .001 & 1.000 & .022 & 22.86 & 4.52 & 4.43 \\
\hline & & .01 & 1.000 & .071 & 7.57 & 14.64 & 13.68 \\
\hline & & .1 & 1.000 & .224 & 2.74 & 49.72 & 40.64 \\
\hline \multirow{12}{*}{2} & \multirow{3}{*}{$\left(\beta_{1}=0.7 ; \beta_{2}=0.8\right)$} & .001 & 1.605 & .029 & 28.86 & 5.76 & 3.53 \\
\hline & & .01 & 1.605 & .090 & 9.52 & 18.51 & 10.92 \\
\hline & & .1 & 1.605 & .281 & 3.46 & 62.67 & 33.23 \\
\hline & \multirow{3}{*}{$\left(\beta_{1}=0.7 ; \beta_{2}=1\right)$} & .001 & 1.572 & .028 & 28.54 & 5.66 & 3.54 \\
\hline & & .01 & 1.572 & .089 & 9.39 & 18.25 & 10.99 \\
\hline & & .1 & 1.572 & .278 & 3.37 & 61.54 & 33.27 \\
\hline & \multirow{3}{*}{$\left(\beta_{1}=1 ; \beta_{2}=1\right)$} & .001 & 1.500 & .027 & 27.89 & 5.53 & 3.62 \\
\hline & & .01 & 1.500 & .087 & 9.16 & 17.82 & 11.23 \\
\hline & & .1 & 1.500 & .273 & 3.26 & 59.86 & 33.76 \\
\hline & \multirow{3}{*}{$\left(\beta_{1}=1 ; \beta_{2}=2\right)$} & .001 & 1.416 & .026 & 27.11 & 5.36 & 3.72 \\
\hline & & .01 & 1.416 & .084 & 8.91 & 17.32 & 11.54 \\
\hline & & .1 & 1.416 & .267 & 3.15 & 58.14 & 34.55 \\
\hline \multirow{12}{*}{3} & \multirow{3}{*}{$\left(\beta_{1}=0.7 ; \beta_{2}=0.8 ; \beta_{3}=1\right)$} & .001 & 1.978 & .032 & 31.95 & 6.44 & 3.20 \\
\hline & & .01 & 1.978 & .100 & 10.44 & 20.49 & 9.86 \\
\hline & & .1 & 1.978 & .315 & 3.64 & 67.95 & 29.63 \\
\hline & \multirow{3}{*}{$\left(\beta_{1}=1 ; \beta_{2}=1 ; \beta_{3}=1\right)$} & .001 & 1.833 & .030 & 30.78 & 6.11 & 3.28 \\
\hline & & .01 & 1.833 & .096 & 10.07 & 19.65 & 10.18 \\
\hline & & .1 & 1.833 & .304 & 3.51 & 65.45 & 30.63 \\
\hline & \multirow{3}{*}{$\left(\beta_{1}=1 ; \beta_{2}=2 ; \beta_{3}=1\right)$} & .001 & 1.726 & .029 & 29.88 & 5.94 & 3.38 \\
\hline & & .01 & 1.726 & .093 & 9.79 & 19.09 & 10.49 \\
\hline & & .1 & 1.726 & .294 & 3.43 & 63.76 & 31.55 \\
\hline & \multirow{3}{*}{$\left(\beta_{1}=1 ; \beta_{2}=2 ; \beta_{3}=2\right)$} & .001 & 1.599 & .028 & 28.78 & 5.71 & 3.51 \\
\hline & & .01 & 1.599 & .089 & 9.44 & 18.39 & 10.89 \\
\hline & & .1 & 1.599 & .283 & 3.33 & 61.57 & 32.72 \\
\hline
\end{tabular}




\section{Conclusões e Considerações Finais}

Neste trabalho pretendeu-se fazer o estudo, na sequência de trabalhos anteriores, de sistemas com componentes em paralelo com diferentes tipos de taxas de risco, considerando a distribuição de Weibull. Esta é tradicionalmente usada neste contexto e noutros, como, por exemplo, na modelação do tempo de incubação do vírus da Sida (HIV1). A introdução de sistemas em paralelo assume relevância quando se pretende aumentar a respectiva fiabilidade.

Apesar de num ponto anterior se terem feito, à medida que os resultados numéricos iam sendo apresentados, as análises pertinentes, vamos agora fazer a síntese dos pontos que nos parecem ser mais significativos. Assim:

a) Introduzindo um novo componente em paralelo num sistema já existente, o tempo médio de vida do sistema global resultante aumenta. Sendo assim, através de uma expressão simples que introduzimos, é fácil concluir que pode diminuir o período de inspecção que minimiza o custo total médio por ciclo (de maior duração) do novo sistema, bem como estimar as condições em que tal pode acontecer. De facto, aumentando a fiabilidade, poder-se-ia pensar que o período de inspecção aumentaria também, de uma forma imperativa, o que não acontece.

b) As distribuições dos tempos de vida dos diversos componentes assumem um papel importante nos diversos resultados que se obtêm. Em particular, tal acontece na precisão das duas aproximações consideradas para o período de inspecção.

c) À medida que o número de componentes em paralelo aumenta, aumentando a fiabilidade do sistema, verifica-se que o tempo médio de detecção se aproxima de metade do período de inspecção. Este facto é relevante ao nível das aproximações consideradas.

d) Podendo, de um modo geral, em termos dos respectivos erros relativos, considerar-se como bastante boas (em certos casos, quase óptimas) as aproximações para o período de inspecção, a verdade é que, em termos dos custos totais médios por ciclo que lhe estão associados, elas podem ainda ser consideradas melhores. Este facto deve-se a que existe uma fraca sensibilidade dos custos totais médios em relação aos períodos de inspecção, conforme, de resto, já tinha sido constatado em outras situações.

e) O custo total médio mínimo por unidade de tempo depende também do número e da distribuição dos tempos de vida dos componentes.

Apesar de tal não ter sido explicitamente referido, é claro que alguns resultados obtidos apontam num sentido diferente do que se constata no caso de sistemas com componentes em série. Por outro lado, considerou-se aqui que o tempo médio de vida dos componentes era igual, o que pode usualmente não acontecer. Por outro lado, finalmente, ao analisar custos médios por unidade de tempo de duração média de um ciclo, consideraram-se valores de custos em situações diferentes. Estes vários aspectos parecem ser merecedores de uma atenção cuidada.

\section{Referências Bibliográficas}

(1) Almeida, C.P.L. (1998). Influência do Período de Inspecção nos Custos de Funcionamento de um Sistema. Tese de Mestrado, ISEG, Lisboa.

(2) Amaral, J.A.; Rosário, M.B.; Paixão, M.T. (2000). Data and Projections of HIV and AIDS in Portugal. Journal of Applied Statistics, 27(3), 269-279. 
(3) Barlow, R.E. \& Proschan, F. (1965). Mathematical Theory of Reliability. Wiley, New York.

(4) Brookmeyer, R. \& Goedert, J.J. (1989). Censoring in an Epidemic With Application to Hemophilia-Associated AIDS. Biometrics, 45, 325-335.

(5) Munford, A.G. \& Shahani, A.K. (1972). A Nearly Optimal Inspection Policy. Operational Research Quarterly, 23(3), 373-379.

(6) Nakagawa, T. \& Yasui, K. (1979). Approximate Calculation of Inspection Policy with Weibull Failure Times. IEEE Transations on Reliabilty, 28(5), 403-404.

(7) Rodrigues Dias, J. (1983). Influence de la Période d' Inspection sur les Coûts dans l' Inspection Périodique de Systèmes. Revue de Statistique Appliquée, 31(4), 5-15.

(8) Rodrigues Dias, J. (1987). Políticas de Inspecção de Sistemas. Tese de Doutoramento, Universidade de Évora.

(9) Rodrigues Dias, J. (1990a). A New Approximation for the Inspection Period of Systems with Different Failure Rates. EJOR - European Journal of Operational Research, 45, 219-223.

(10) Rodrigues Dias, J. (1990b). Some Approximate Inspection Policies for a System with Imperfect Inspections. Recherche Opérationnelle / Operations Research, 24(2), 191-199.

(11) Rodrigues Dias, J. (2000a). Análise do Período de Inspecção de um Sistema com Componentes em Série e Tempos e Vida Exponenciais. Ingenium, II Série, 51, 86-88.

(12) Rodrigues Dias, J. (2000b). Importância de uma Rápida Obtenção de Dados na Redução de Custos em Controlo Estatístico de Qualidade. Actas das VII Jornadas de Classificação e Análise de Dados, JOCLAD 2000, 76-80.

(13) Rodrigues Dias, J. (2001). Análise de Períodos de Inspecção com Componentes em Paralelo e Tempos de Vida Exponenciais. In: Um Olhar Sobre a Estatística [edited by Pedro Oliveira and Emília Athayde], 594-605.

(14) Rodrigues Dias, J.; Infante, P. (1999). Analisys of the Inspection Period in a System with Serial Components and a Weibull Lifetime Distribution. Actas do IV Congreso Galego de Estatística e Investigación de Operacións, Universidade de Santiago de Compostela, 135-138.

(15) Rodrigues Dias, J.; Infante, P. (2001). Comparação de Períodos de Inspecção e de Custos em Sistemas com Componentes em Série e com Diferentes Tipos de Taxas de Risco. In: Um Olhar Sobre a Estatística [edited by Pedro Oliveira and Emília Athayde], 160-171. 